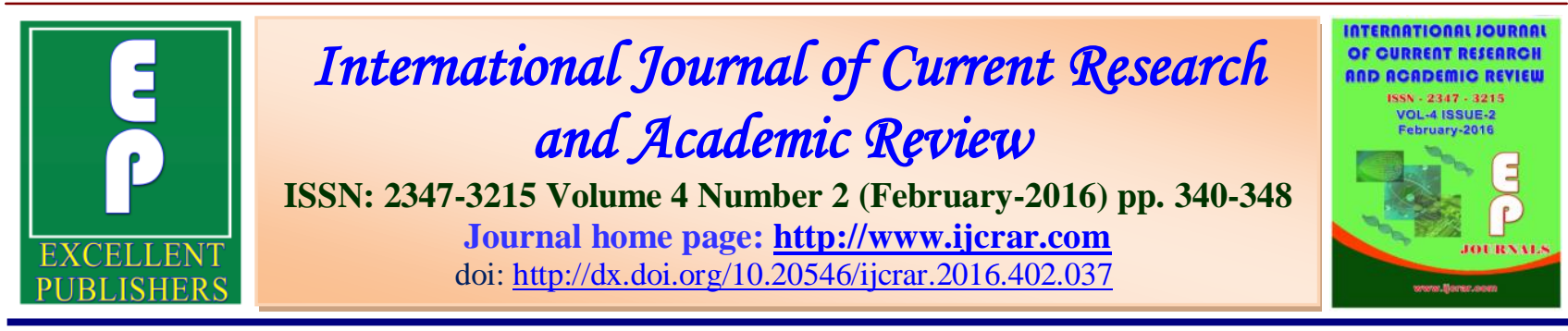

\title{
Ultrasonographic evaluation of peripheral nerves in Diabetic and it's correlation with nerve conduction
}

\section{B. Varadharaju*}

Assistant Professor, Department of Physiology, Meenakshi Medical College and Research Institute, Kanchipuram-631552, Tamil Nadu, India

*Corresponding author

\begin{tabular}{l|l} 
KEYWORDS & A B S T R A C T
\end{tabular}

Cross Sectional Area;

Diabetic Neuropathy;

Our recent studies have shown that to investigate the ultrasonographic

Echo Intensity;

Median Nerve;

Sonography;

Tibial Nerve evaluation of peripheral nerves in diabetic and it's correlation with nerve conduction. All nerve conductions in more than one limb indicates generalized diseased nerves, or generalized peripheral neuropathy. People with diabetes mellitus often develop generalized peripheral neuropathy. Twenty eight with type 2 diabetes (mean \pm SD, $58.2 \pm 6.7$ years) and Twenty fifth healthy volunteers (mean, $57.48 \pm 5.62$ years) were enrolled in this study The cross-sectional area (CSA) and echo intensity of the peripheral nerve were evaluated at the carpal tunnel and proximal to the wrist (wrist) of the median nerve and in the tibial nerve at the ankle. There was a significant increase in the CSA and hypoechoic area of the nerve in diabetic patients compared with controls (wrist, $6.8 \pm 1.2 \mathrm{~mm} 2,60.1 \% \pm 6.0 \%$; ankle, $8.7 \pm$ $1.3 \% \mathrm{~mm} 2,54.6 \% \pm 3.2 \%$; and wrist, $8.9 \pm 2.8 \mathrm{~mm} 2,70.2 \% \pm 7.1 \%$; ankle, $14.0 \pm 5.9 \mathrm{~mm} 2,60.1 \% \pm 6.1 \%$ in controls and diabetic patients, respectively; $\mathrm{P}<.05)$. Cross-sectional areas were negatively correlated with reduced motor nerve conduction velocity and delayed latency. These results suggest that sonographic examinations are useful for the diagnosis of diabetic neuropathy.

\section{Introduction}

The World Health Organization estimates that more than 220 million people worldwide have diabetes mellitus (DM). This figure is estimated to more than double by $2030^{1}$ Advanced DPN causes serious complications, such as diabetic foot ulcers, gangrene, and Charcot joint, all of which worsen the quality of life of diabetic patients $^{2}$. In symptomatic diabetic neuropathy, there is slowing of nerve conduction velocity owing to demyelination and loss of large myelinated fibers, and a decrease in nerve action potentials owing to loss of axons. Changes in conduction 
velocity can be detected in symptomatic patients, but their presence is not predictive of the onset of symptomatic neuropathy. ${ }^{3}$

Nerve conduction studies (NCS) are the most objective noninvasive measures of nerve function. They represent a valuable tool of evaluation of neuropathy in large clinical and epidemiological studies NCS should not be considered a substitute for careful clinical examination, because NCS have many pitfalls and their results must be interpreted in the context of clinical data.The test is highly sensitive and its not invasive, but can be a little painful due to the electrical shocks. The shocks are associated with a low amount of electrical current so they are not dangerous to anyone. Who already has an electrical device in body. The test takes longer time to perform ${ }^{4}$.

The duration of diabetes and poor glycemic control proved to be more important risk factors over $5 \mathrm{yr}$ as related to the development of subclinical neuropathy. Therefore it can be used as a screening tool for asymptomatic diabetics ${ }^{5,6}$.

A nerve conduction study is complex, but, in general, different pathological processes result in changes in latencies, motor, and/or sensory amplitudes, or slowing of the conduction velocities to differing degrees. If a response is much slower than normal, damage to the myelin sheath is implied. If the nerve's response to stimulation by the current is decreased but with a relatively normal speed of conduction, damage to the nerve axon is implied ${ }^{7,8}$.

\section{Materials and Methods}

\section{Chemicals}

Glucose kits and HbA1C were purchased from immune Diagnostic kits, USA and All the other chemicals used were of analytical grade.

\section{Sonographic Examinations}

Sonographic examinations was performed by the ultrasound equipment PHILIPS HD 11 with a multi-frequency linear probe of 7.5 to $12 \mathrm{MHz}$

\section{Ethical Concern}

Ethical clearance was obtained from the Ethical committee meeting conducted at Meenakshi Medical College and Hospital.

\section{Statistical Analysis}

Data were analyzed using the SPSS software package, version 17.0 (SPSS Inc., Chicago, Illinois, USA). Quantitative data were expressed using range, mean, SD, and median, whereas qualitative data were expressed as frequency and percentage. Qualitative data were analyzed using the $\chi^{2}$ test; also, exact tests such as Fisher's exact were used to compare the two groups. Nonnormally distributed quantitative data were analyzed using the Mann-Whitney test to compare the two groups. The Pearson coefficient was used to analyze the correlation between any two variables. $P$ value was assumed to be statistically significant at 0.05 .

\section{Experimental Design}

Twenty eight patients with type 2 diabetes with neuropathy were enrolled in this study at the Meenakshi Medical College and Research Institute, Kanchipuram (20 men and 8 women; age range, 40-69 years; mean $58.24 \pm 6.709$ ). Our control group consisted of 25 healthy volunteers without diabetes mellitus or CTS (14 men and 11 women; age range, 48-69 years; mean, $57.48 \pm$ $5.620)$. 
Every participant was able to walk unaided, and none had received hemodialysis.

All the participants gave their full consent. Their physical measurements like height, weight, BMI etc. were calculated. They had their blood biochemical tests for glucose (fasting and post prandial, random ), HbAc1. Later all of them had ultrasound examination done in both the wrists. Some had their tibial nerve ultrasonography. A standard protocol was maintained for USG. Then all these patients were subjected to nerve conduction studies.

We studied a total of $126(56+50+15+5)$ peripheral nerves (including 106 median nerves and 20 tibial nerves) of 53 participants who had both sonography and NCS done.

All participants were in the supine position on a table with fingers semi ex-tended during examination of the median nerve and in the prone position during examination of the tibial nerve. The volar wrist crease and pisiform bone or medial malleolus were used as initial external reference points and landmarks during scanning. Transverse and longitudinal sonograms of the nerve at each position were recorded

\section{Results and Discussion}

Early carpal tunnel syndrome in which sonographic findings of bowing of the carpal tunnel has not yet set in might be mistaken as part of diabetic peripheral neuropathy (Figure 1- A, B, C, D). The peripheral nerve's speckled pattern on sonography enabled us to assess its size and echo intensity.

Figure. 2. Shows that the normal appearance of the peripheral nerve can be readily recognized. The nerve consists of multiple hypoechoic bands corresponding to neuronal fascicles, which are separated by hyperechoic lines that correspond to the epineurium.

The prevalence of diabetes for all agegroups worldwide was estimated to be $2.8 \%$ in 2000 and projected to be $4.4 \%$ in 2030 . The total number of people with diabetes is projected to rise from 171 million in 2000 to 366 million in 2030. The most important demographic change to diabetes prevalence across the world appears to be the increase in the proportion of people $>65$ years of age ${ }^{9}$.

Diabetes Mellites is becoming a major cause of premature disability in India and peripheral neuropathy is a common complication ,sometimes even the presenting symptom, of Diabetes mellites ${ }^{10}$, The diagnosis of neuropathy is based mainly on the characteristic symptoms and signs, confirmed with NCS, till date. However the NCS are time-consuming, slightly invasive and generally not well tolerated for repeated evaluation. On the other hand USG studies are non-invasive, cost effective and can be done quickly, even as a bed side procedure..They are also useful alternatives to NCS with high sensitivity and specificity.

The goal of the investigation of peripheral neuropathy is to establish the diagnosis, determine whether it is an axonal or demyelinating process and to find its cause.

Clinically neuropathy causes weakness and atrophy of muscle, loss of sensation ( like pain, temperature, touch and vibration) or altered sensation ( pain, parasthesia, etc) and weak or absent tendon reflexes.

Careful history taking with attention to family and personal history, environmental exposure ,systemic illness, combined with a thorough neurological examination and 
Int.J.Curr.Res.Aca.Rev.2016; 4(2): 340-348

relevant laboratory studies can determine the etiology in most peripheral neuropathies.

Nerve conduction studies (N.C.S.) can distinquish demyelinative neuropathy (slowing of conduction velocity or conduction block) from axonal neuropathy (low action potential amplitudes).

Electromyography (E.M.G.) can distinguish enervation atrophy from primary muscle disease.

The Pressure- Specified -Sensory Device testing provides a clinical measurement of loss of sensory loss of sensory discrimination in chronic peripheral neuropathies. This test is limited ,if used alone, as it does not discriminate focal disease (e.g. entrapement neuropathies) from diffuse abnormality of the affected nerve (e.g. diabetic neuropathy). The effectiveness of this device increases dramatically when combined with ultrasongraphic studies. Such a combined study helps plan effective treatment strategy, possibility of early surgical intervention leading to improvement in patient symptoms

When the diagnosis is in doubt a nerve biopsy can give more definitive information. Sometimes a skin biopsy may show altered density of small nerve fibres in epidermis.

DPN predominantly affect small myelinated and unmyelinated fibers that convey sensation like pain and temperature. Degeneration such small fibers involve the most distal portion of the nerve fibers.NCS and EMG may be normal in such cases and sural nerve biopsy may be difficult to interpret.
Conventional motor and sensory NCS has been widely used to diagnose DPN. Symptoms of DPN appear bilaterally from the toes or the soles of the feet. Thus, NCS in the lower limbs should be more suitable to assess DPN severity. However, NCS in the lower limbs is time-consuming, and the action potential in the lower limbs sometimes cannot be evoked in cases of patients with advanced DPN. Some previous studies have reported that nerve conduction velocity slowing in the upper limbs is similar to that in the lower limbs of diabetic patients. Further Skin temperature and humidity, easily affect the sensory nerve conduction velocity studies at the time of measurement. Mizumoto et al chose to look at distal motor latency and the MCV only because the sensory nerve conduction velocity were not measurable in many patients and appeared to be an unsuitable parameter. For the above reasons, we also performed only the motor nerve conduction study, especially the NCV and latency of median nerve were measured.

The use of diagnostic ultrasound for peripheral nerve lesions is becoming more prevalent nowadays as the related hardware, software and techniques in ultrasonography improve tremendously. Several recent articles focus on the use of ultrasound in diagnosing or mapping peripheral nerve injuries $^{11}$. Even in animal studies the correlation between anatomical measurements and ultrasound measurements have been established. But there are only few previous studies found ,both in Indian and world literature, focusing on monitoring or documenting peripheral nerve lesions in diabetic peripheral neuropathy using diagnostic ultrasonography. 
Int.J.Curr.Res.Aca.Rev.2016; 4(2): 340-348

Fig.1A Median Nerve in LAX

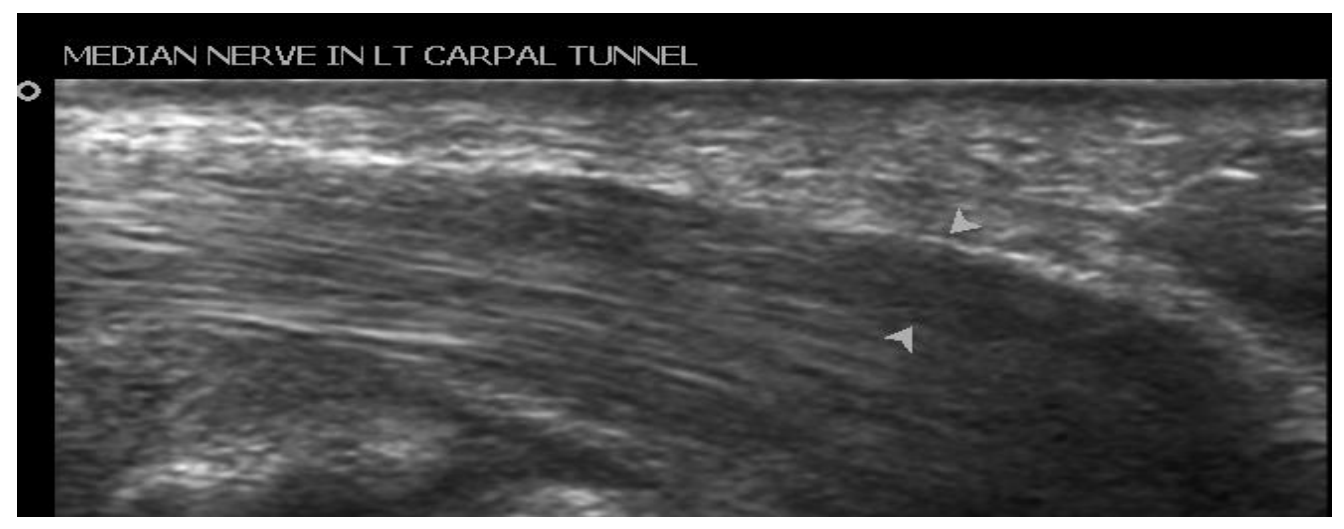

Fig.1B Median Nerve in SAX

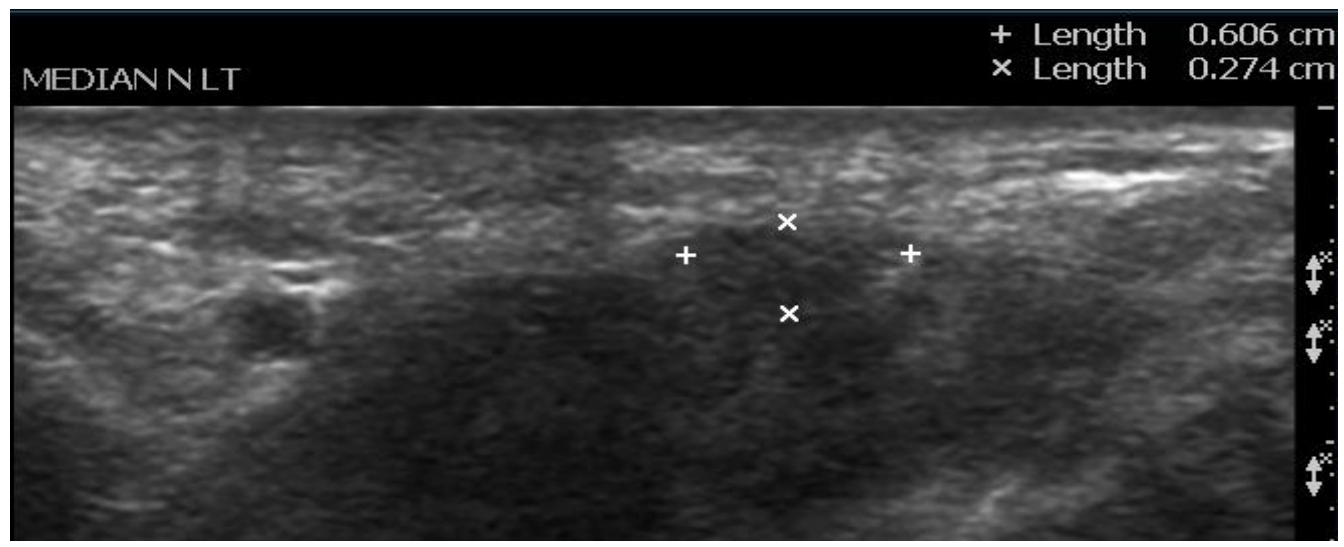

Fig.1C Tibial Nerve in LAX

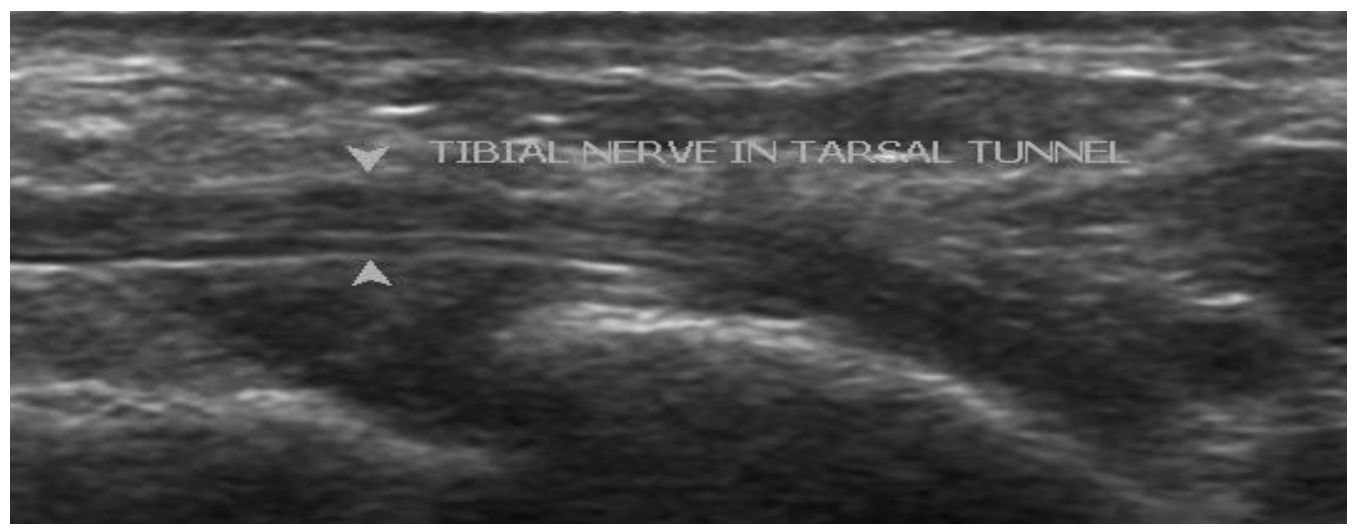


Int.J.Curr.Res.Aca.Rev.2016; 4(2): 340-348

Fig.1D Tibial Nerve in SAX

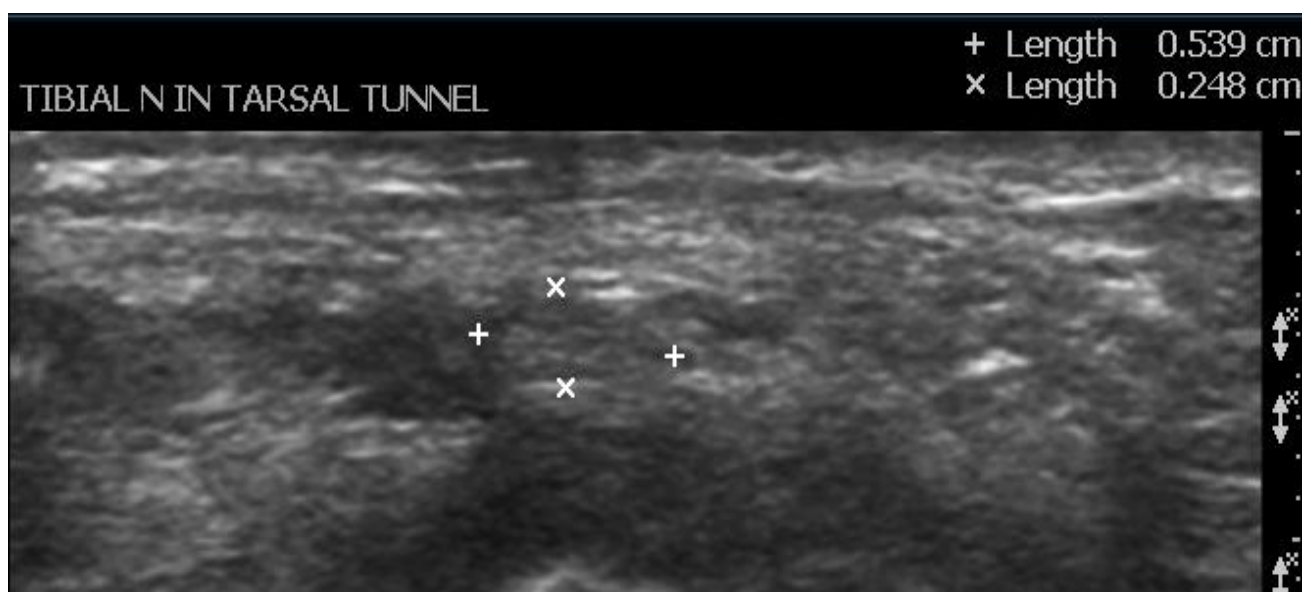

Fig.2 Median Nerve in Carpal Tunnel Showing the Typical Cross-Sectional Appearnance (White Arrow)

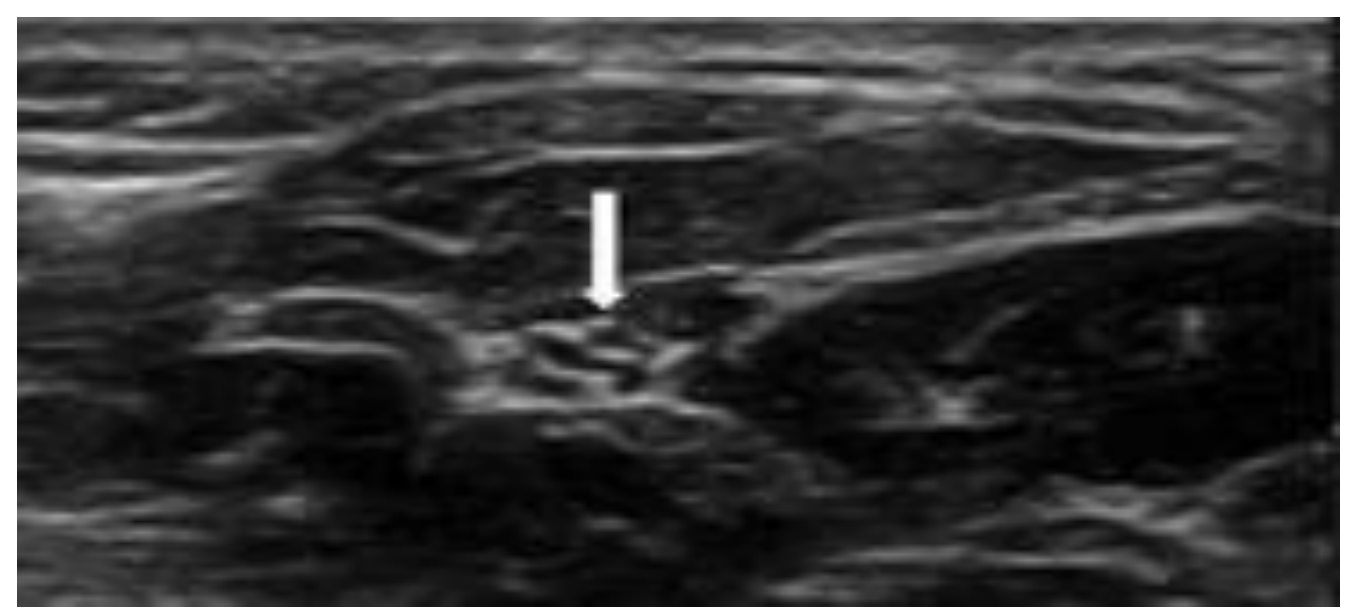

Fig.3 SAX of Median Nerve in Control ( A ) in Diabetic Patient With DPN ( B)
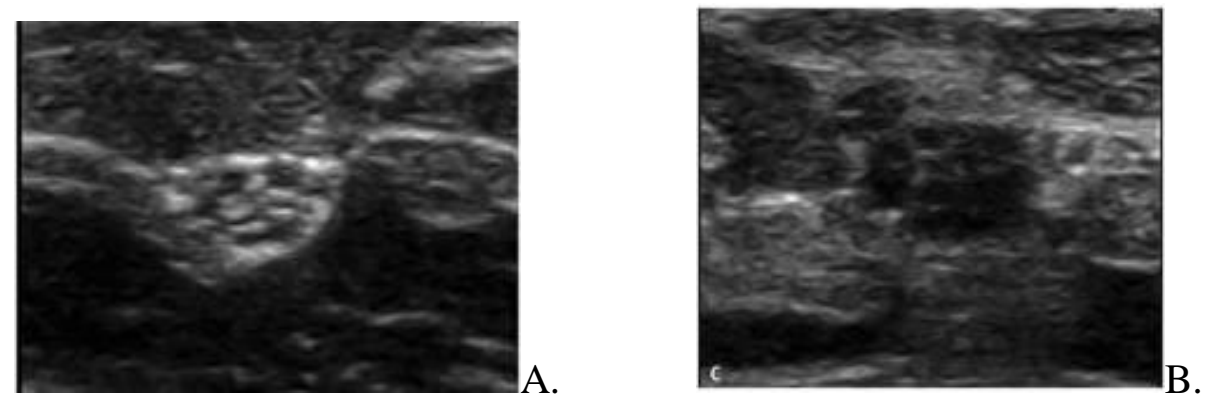
Fig.4 SAX of Tibial Nerve in Control ( A ) in Diabetic Patient With DPN ( B)

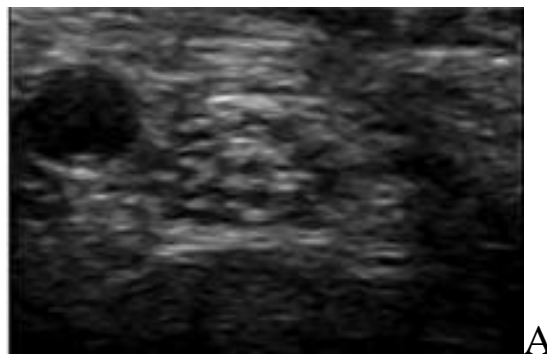

Sonographic criteria for the diagnosis of neuropathy have been proposed by several studies and confirmed in our study also. We found that the CSA of both median and tibial nerves in diabetic patients were significantly larger than those in controls. Carpal tunnel syndrome and tarsal tunnel syndrome (TTS) are the most common entrapment neuropathies that can occur in diabetics.

Several studies have shown that the CSA of median and tibial nerves were significantly larger in all diabetic patients with polyneuropathy than in controls. Our study has also confirmed this finding. It has also been shown that some diabetic patients without neuropathy also have significant increase in CSA

Of the two songraphic measurements of nerve C.S.A, we used the easier reproducible method. Further multilevel assessment of the median nerve has been suggested by some authors as there are individual variations in the swollen part of the median nerve. We studied the median CSA with reference to the fixed bony landmark ( the pisiform bone ) in all cases.

Using sonography in the median nerve, Sernik et al showed decreased echogenicity of the median nerve in symptomatic CTS wrists. This has been shown even in tibial nerves. Kim et al reported that $6.8 \%$ of diabetic patients had asymptomatic electrophysiologic $\mathrm{CTS}^{10,12}$.

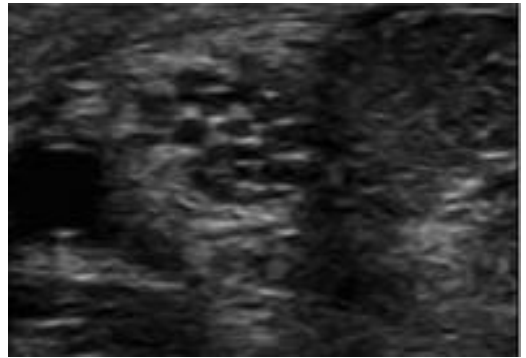

B

Therefore asymptomatic patients can also be evaluated by USG for presence of increased CSA., which may precede the clinical manifestations. Thus USG can be tried as screening procedure for pre-clinical diagnosis of polyneuropathy in all cases of chronic Diabetes Mellites.

It has been hypothesized that in DM patients the peripheral nerves are swollen because of increased water content related to increased aldose reductase conversion of glucose to sorbitol, which in turn contributes to increased intracellular hydration.( This has been confirmed even by MRI studies ). These histo-chemical changes contribute to changes in NCS and morphological changes seen in USG. The nerve swelling is responsible for increased CSA in USG and slowing of conduction in NCS.

There are some limitations in our study, the relatively small patient study group.,the lack of normative data for the common peroneal nerve, tibial, sural, nerve size in lower limb, and median, ulnar nerve size. in upper limb in non-diabetics and in diabetics without neuropathy in Indian population. The other limitations of the application of highresolution USG to the evaluation of the peripheral nerves, are its operator dependence and its relatively long learning curve.In experienced hands high-resolution USG is an very effective imaging modality for capturing morphologic changes in the peripheral nerves and for making correct 
diagnoses. It could be used either as a firstline imaging modality in patients with suspected peripheral nerve lesions before further treatment is administered or as a useful tool for follow-up imaging or as a screening tool in asymptomatic diabetic patients.

\section{Conclusion}

Thus sonographic examinations are very useful for the early diagnosis of diabetic neuropathy. Early detection of nerve dysfunction is important to provide appropriate care for patients with diabetic polyneuropathy. The entrapment neuropathies are always progressive, if not addressed early, they can become permanent and may lead to irreparable tissue damage and its attended complications. Timely medical and surgical interventions can cause restoration of sensation and relief of pain. Therefore early diagnosis of these neuropathies becomes very important. Advances in sonographic technology, like color Doppler, power Doppler, tissue harmonic imaging, elastography, etc. allows more accurate imaging of peripheral nerves of the upper and lower limbs. The high sensitivity and specificity of USG makes it a suitable, cost-effective reproducible diagnostic modality in all cases of symptomatic and asymptomatic diabetic patients.

Continual technologic improvements, including the availability of high-frequency transducers (6-13-MHz) will lead to an increase in the use of sonography in the imaging of peripheral nerves. We believe that sonography should be the primary technique for imaging peripheral nerve pathology because it is inexpensive and widely available, has no contraindications, and allows rapid, detailed imaging of the entire length of the major peripheral nerves of both limbs.

\section{References}

1. Wild, S.; Roglic, G.; Green, A.; Sicree, R.; King, H. (2004). Global prevalence of diabetes: estimates for the year 2000 and projections for 2030. Diabetes Care 2004 ; 27: 1047-1053.

2. Ogawa, K.; Sasaki, H.; Yamasaki, H.; Okamoto, K.; Matsuno, S.; Shono, T.; Doi, T.; Arimoto, K.; Furuta, H.; Nishi, M.; Nakao, T.; Nanjo, K. Peripheral nerve functions may deteriorate parallel to the progression of microangiopathy in diabetic patients.Nutr Metab Cardiovasc Dis 2006; 16 : 313-321.

3. Chudzik, W.; Kaczorowska, B.; Przybyła, M.; Chudzik, B.; Gałka, M. Diabetic neuropathy. Pol Merkur Lekarski 2007; 22: 66-69.

4. Colak, A.; Kutlay, M.; Pekkafali, Z.; Saraçoglu, M.; Demircan, N.; Simşek, H.; Akin, ON. Kibici, K. Use of sonography in carpal tunnel syndrome surgery: a prospective study. Neurol Med Chir 2007; 47: 109-115.

5. Wiesler, ER.; Chloros, GD.; Cartwright, MS.; Smith, BP.; Rushing, J.;Walker, FO. The use of diagnostic ultrasound in carpal tunnel syndrome. J Hand Surg Am 2006;31: 726-732.

6. Watanabe, T.; Ito, H.; Sekine, A.; Katano, Y.; Nishimura, T.; Kawase, H.; Kato, Y.; Matsuoka, T.; Takeda, J.; Seishima, M. Sonographic evaluation of the peripheral nerve in diabetic patients: the relationship between nerve conduction studies, echo intensity, and cross-sectional area. J Ultrasound Med 2010; 29: 697-708.

7. Said, G. Diabetic neuropathy: a review. Nat Clin Pract Neurol 2007;3:331-340. 
8. Yagihashi, S.; Yamagishi, S.; Wada, R. Pathology and pathogenetic mechanism of diabetic neuropathy: correlation with clinical signs and symptoms. Diabetes Res Clin Pract 2007; 77: 184-189.

9. Mizumoto D, Hashizume H, Senda M, Nagoshi $M, \quad$ Inoue $H$. Electrophysiological assessment of the carpal tunnel syndrome in hemodialysis patients: formula for predicting surgical results. J Orthop Sci 2003; 8:8-15.

10. Kim WK, Kwon SH, Lee SH, Sunwoo I. Asymptomatic electrophysiologic carpal tunnel syndrome in diabetics: entrapment or polyneuropathy. Yonsei Med J 2000; 41:123-127.

11. Cartwright MS, Passmore LV, Yoon JS, Brown ME, Caress JB, Walker FO. Cross-sectional area reference value for nerve ultrasonography. Muscle Nerve 2008; 37:566-571.

12. Severinsen K, Andersen H. Evaluation of atrophy of foot muscles in diabetic neuropathy: a comparative study of nerve conduction studies and ultrasonography. Clin Neurophysiol 2007; 118:2172-2175.

\section{How to cite this article:}

Varadharaju, B., 2016. Ultrasonographic evaluation of peripheral nerves in Diabetic and it's correlation with nerve conduction. Int.J.Curr.Res.Aca.Rev. 4(2): 340-348. doi: http://dx.doi.org/10.20546/ijcrar.2016.402.037 